\title{
The short-term effects of laparoscopic sleeve gastrectomy on hematological parameters
}

\author{
(1) Fatih Can Karaca \\ Department of Health Sciences, Bilgi University, İstanbul, Turkey
}

\begin{abstract}
Introduction: Sleeve gastrectomy (SG) surgery is an effective method for weight loss in obese individuals. The data on the effect of this surgical intervention on iron metabolism and hematological factors are limited. This study aimed to investigate the effects of SG surgery on iron and red blood cell-related parameters.

Materials and Methods: The six-month follow-up data of 33 patients who underwent SG for obesity treatment were evaluated retrospectively in this study. Whole blood levels of hemoglobin, mean corpuscular volume (MCV), mean corpuscular hemoglobin concentration (MCHC), red cell distribution width (RDW-CV), and serum iron concentrations were evaluated.

Results: Mean hemoglobin, $\mathrm{MCHC}$, and RDCV levels did not show a significant difference compared to the baseline and between months. Mean MCV levels significantly differed from baseline values at the end of the third month. Serum iron levels were significantly higher on the 6-month follow-up visit.

Conclusion: The findings suggest that the iron level and other iron-related hematological parameters of the patients who underwent SG improved after the surgery.

Keywords: Anemia; iron deficiency; sleeve gastrectomy.
\end{abstract}

\section{Introduction}

According to the World Health Organization, the prevalence of obesity is increasing every year, becoming a pandemic. ${ }^{[1]}$ Besides decreasing the life quality and self-esteem of patients, obesity is closely related to several chronic conditions, including cardiovascular diseases and type 2 diabetes. ${ }^{[2]}$ Among the treatment options for obesity and obesity-related complications, bariatric surgery procedures are evaluated as the most effective treatment for severe obesity, and the number of surgery cases follows an increasing trend recently. ${ }^{[3]}$

Laparoscopic Sleeve Gastrectomy (SG) is a procedure sug- gested for obese individuals, as a result of lower mortality and morbidity rate compared to other bariatric surgery procedures such as biliopancreatic diversion and gastric by-pass surgery. ${ }^{[4-6]}$ In the SG, the 60 to $80 \%$ of the stomach is removed, leaving the stomach in a tubular shape, thus limiting the intake of excess food and increasing satiety, resulting in rapid weight loss. Besides, most of the patients experience resolution or improvement of comorbidities after the procedure. ${ }^{[7]}$

There are studies that show an increased ratio of anemia and iron deficiency after bariatric surgery, due to insufficient absorption of the nutrients and iron in the duo- 
denum. Besides iron, folic acid, Vitamin B12, Vitamin D absorption might also get affected. ${ }^{[8]}$

With the SG approach, transforming a part of the gastrointestinal tract might still cause nutritional disturbances, and the nutritional status of the patients must be assessed after all types of bariatric surgery procedures. Furthermore, there is no consensus on the amount and duration of iron supplementation for these patients.

The aim of this retrospective study was to investigate the presence of any iron deficiency and anemia after SG during within an early follow-up period of six months.

\section{Materials and Methods}

We conducted a retrospective study comparing preoperative and early postoperative hematological parameters including hemoglobin, mean corpuscular volume (MCV), mean corpuscular hemoglobin concentration (MCHC), red cell distribution width (RDW-CV) and iron levels in patients who underwent SG.

Data collection and analysis was performed in compliance with the Helsinki Declaration, and informed consent was taken from all participants.

Routine preoperative examinations were done by an expert cardiologist, endocrinologist, anesthesiologist, ophthalmologist, neurologist, and psychiatrist team. Any sign of malignity was excluded using proper diagnostic tools. All patients fulfilled the criteria for the SG surgery and were with a body mass index (BMI) of $>35$, or $>40 \mathrm{~kg} /$ $\mathrm{m}^{2}$, corresponding to Class II and Class III obesity.

SG was performed in a $30^{\circ}$ reverse Trendelenburg position using the laparoscopic technique as described by Braghetto et al. ${ }^{[9]}$ Operation was performed by a single surgeon experienced in bariatric and metabolic surgery procedures. None of the patients required a conversion from laparoscopy to laparotomy in the perioperative period.

Blood samples were obtained in the follow-up visits on the first, third, and sixth months after the procedure and analyzed using automatized hematology and clinical chemistry analyzers.

We collected data on weight loss and laboratory results of specific parameters.

Of all patients with hemoglobin concentrations $<12 \mathrm{~g} / \mathrm{dL}$ were classified as having anemia.

We did not prescribe iron supplementation for any of the patients. The patients received a multivitamin B supplementation, including the vitamins B6, B9, and B12 during the follow-up period.

\section{Statistical Analysis}

The data were presented as mean values and standard deviation.

Paired sample t-test and nonparametric Wilcoxon SignedRank test were used for the analyses. Statistical analyses were performed for each follow-up analysis versus pre-operative values and other follow-up results. A significance level of $\mathrm{p}<0.05$ was adopted for the values. GraphPad Prism 7.0 software for Windows (California, USA) were used for statistical analyses.

\section{Results}

Thirty-three patients who completed a postoperative follow-up of 6 months were selected for the evaluation. Twenty-seven of them were in female and six were male with a mean age of $51.5 \pm 10.7$. The mean baseline BMI of the female patients was $45.54 \pm 4.45$, whereas $51.74 \pm 2.87 \mathrm{~kg} / \mathrm{m}^{2}$ for male patients. The 6-month follow-up rate was $93 \%$. The mean weight loss (\%WL) for the analyzed 33 patients was $10.1 \% \pm 1.24 \%, 21.4 \% \pm 2.27 \%$, and $32.6 \% \pm 3.04 \%$ at 1 month, 3 months, and 6 months, respectively. At the end of the follow-up, there was a significant reduction in mean BMI values. Postoperative course of the patients was uneventful.

The follow-up results of the hematological parameters were shown in Table 1. Mean hemoglobin, MCHC, and RDW-CV levels did not show a significant difference between the groups. Mean MCV levels significantly differed from baseline values at the end of the third month in an increasing trend $(p<0.05)$. Serum Iron levels were significantly higher on the 6-month follow-up visits when compared to the baseline, first, and third-month follow-up visit values ( $p<0.05$ for all comparisons).

\section{Discussion}

Bariatric surgery procedures gaining more importance and demand as the ratio of obese individuals increase rapidly worldwide. Among the other bariatric procedures, SG is a more beneficial and effective option for weight loss in obese compared to other operative methods. In comparison to biliopancreatic diversion and gastric by-pass, SG does not require intestinal anastomoses, and change in the gastrointestinal tract is minimum. 
Table 1. Hematological variables in the preoperative and postoperative follow-up period

\begin{tabular}{|c|c|c|c|c|c|c|c|c|c|}
\hline & \multicolumn{2}{|c|}{ Baseline } & \multicolumn{2}{|c|}{$1^{\text {st }}$ month } & \multicolumn{2}{|c|}{$3^{\text {rd }}$ month } & \multicolumn{2}{|c|}{$6^{\text {th }}$ month } & \multirow[t]{2}{*}{ p* } \\
\hline & Mean & SD & Mean & SD & Mean & SD & Mean & SD & \\
\hline Hemoglobin $(\mathrm{g} / \mathrm{dL})$ & 13.01 & 1.55 & 13.37 & 1.17 & 13.61 & 1.42 & 13.82 & 1.77 & NS \\
\hline $\operatorname{MCV}(\mathrm{fL})$ & 83.27 & 5.41 & 85.08 & 3.70 & 88.02 & 6.06 & 84.55 & 3.45 & $<0.05 ¥$ \\
\hline $\mathrm{MCHC}(\mathrm{g} / \mathrm{dL})$ & 33.25 & 1.49 & 33.38 & 0.93 & 33.56 & 1.10 & 33.98 & 2.10 & NS \\
\hline RDW-CV (\%) & 26.75 & 20.50 & 21.50 & 16.12 & 19.52 & 14.83 & 14.18 & 1.91 & NS \\
\hline Iron (mcg/dL) & 62.71 & 34.21 & 53.57 & 17.73 & 71.23 & 24.71 & 89.4 & 39.83 & $<0.05 f$ \\
\hline
\end{tabular}

*p value was calculated compared to the prior visits; ¥ Significant difference between the baseline and third month follow-up visit results; $€$ Significant difference between the baseline and sixth month, first and sixth month, and third and sixth month follow-up visit results; SD: Standard deviation; MCV: Mean corpuscular volume; MCHC: Mean corpuscular hemoglobin concentration; RDW-CV: Red cell distribution width.

We carried out a retrospective study of 33 individuals who underwent SG for weight loss and evaluated the hematological outcomes after the surgery during a six-month early follow-up duration. On the initial 6-month follow-up period, the entire patient population experienced weight loss. None of the patients had a gain of lost weight or required re-operation for surgery-related comorbidities.

Short and long term complications after bariatric procedures include nutritional deficiencies alongside others, including leakage from the stapler site, gastroesophageal reflux, and gastrointestinal complaints. Studies reporting nutritional deficiency cases focusing on iron, vitamin B12, and Vitamin D were also shown in the literature. ${ }^{[8]}$ There are studies reporting increased rates of iron deficiency anemia for patients who underwent bariatric procedures such as biliopancreatic diversion and gastric by-pass, and nutritional deficiencies and hematological status of the patients after SG were rarely evaluated. ${ }^{[10]}$

The most common type of anemia worldwide is iron deficiency anemia. Anemia diagnosis is made by evaluating hematological variables including, complete blood count parameters, iron, ferritin, total iron-binding capacity, and transferrin saturation. According to WHO, the diagnosis of anemia is made when $\mathrm{Hb}$ concentration is below $13 \mathrm{~g} /$ $\mathrm{dL}$ for male, and below $12 \mathrm{~g} / \mathrm{dL}$ for females aged 15 years or above. ${ }^{[1]}$

Although SG did not change the anatomy of the intestinal tract and duodenum, where iron is mainly absorbed, iron uptake still can be affected from the reduced gastric volume, as it requires gastric acid for the reduction of non-haem iron to absorbable form. ${ }^{[12]}$ Absorption of iron is induced or inhibited by the numerous diet-related and individual factors such as ingested type of the iron (heme or non-heme), intestinal factors, and demands of the organism. Heme iron from nutrients is released from hemoglobin and myoglobin and absorbed by the enterocytes on the duodenum and proximal jejunum. Reducing agents such as ascorbic acid and gastric hydrochloric acid are required for the release and absorption of heme iron. ${ }^{[13]}$

Gastrointestinal complications after bariatric surgery, including diarrhea, gastroesophageal reflux thus causing using of proton pump inhibitors results in inadequate iron absorption. ${ }^{[14]}$ Furthermore, the release of iron from myoglobin and hemoglobin after ingestion of red meat is affected due to gastrointestinal alterations and reduces the bioavailability of dietary iron. ${ }^{[15]} \mathrm{A}$ decrease in intake and absorption of the iron, increased need for iron, especially for women of reproductive age results in diminished hemoglobin synthesis and iron deficiency anemia. Anemia results in a deficiency in oxygen transport to the organs and cells, resulting in impaired physical tolerance and fatigue.

Although studies are presenting different rates of anemia and iron deficiency following bariatric surgery, the controversy among the studies might be a result of different risk factors among the individuals. After evaluating the iron and hematological status of our study group, we suggest that there is no need for iron supplementation after SG. The improved iron and MCV status might be an outcome of a better-established diet following the surgery. It is a fact that obese individuals often have an inadequate diet rich in fats and carbohydrates, which is poor of required micro and macronutrients. Therefore, a well-tailored diet by a dietician might be related to the improved hematological parameters in our study group. On the other hand, increased MCV levels might suggest 
a Vitamin B12 deficiency following the surgery, we did not experience decreased levels of this vitamin in our patients. Furthermore, it was reported that obesity causes an inflammatory state and increases the cytokine levels in the organism. Immune system activation reduces plasma iron levels through increased hepcidin production. ${ }^{[16,17]}$ Thus, increased iron levels in our subjects after SG might be possibly due to decreased inflammatory status in the organism. However, hematological parameters should be closely monitored alongside the metabolic variables in the follow-up visits, and any sign of nutritional decline should be overcome.

It has been suggested that nutritional parameters should be evaluated once a year during the life course, even after the patient's weight stabilizes. ${ }^{[8]}$ Although some reports suggest vitamin supplementation after the surgery, there is no data regarding the nutritional needs of patients who underwent a bariatric surgery procedure.

A limitation of this study is that we did not analyze serum ferritin levels as an indicator of iron status. However, being an acute phase reactant, ferritin levels would be increased in patients with an inflammatory condition, and yield false-negative results for the patients with iron deficiency.

In conclusion, we suggest that SG is not associated with anemia and iron deficiency in our study group. Further studies with the addition of different hematological markers are required in order to evaluate the hematological status of patients who underwent SG. Improved hematological parameters may be an outcome of the better nutritional status and decreased the inflammatory state of the organism.

\section{Disclosures}

Ethichs Committee Approval: The Ethics Committee of Health Sciences University, Istanbul Training and Research Hospital provided the ethics committee approval for this study.

Peer-review: Externally peer-reviewed.

Conflict of Interest: None declared.

\section{References}

1. WHO. Obesity and overweight. Available at: https://www. who.int/en/news-room/fact-sheets/detail/obesity-andoverweight. Accessed Mar 27, 2020.

2. Nyberg ST, Batty GD, Pentti J, Virtanen $M$, Alfredsson L, Fransson El, et al. Obesity and loss of disease-free years owing to major non-communicable diseases: a multicohort study. Lancet Public Health 2018;3:e490-e7. [CrossRef]

3. Angrisani L, Santonicola A, lovino P, Vitiello A, Higa K, Himpens J, et al. IFSO Worldwide Survey 2016: Primary, Endoluminal, and Revisional Procedures. Obes Surg 2018;28:3783-94.

4. Deitel M, Gagner M, Erickson AL, Crosby RD. Third International Summit: Current status of sleeve gastrectomy. Surg Obes Relat Dis 2011;7:749-59. [CrossRef]

5. Kikkas EM, Sillakivi T, Suumann J, Kirsimägi Ü, Tikk T, Värk PR. Five-Year Outcome of Laparoscopic Sleeve Gastrectomy, Resolution of Comorbidities, and Risk for Cumulative Nutritional Deficiencies. Scand J Surg 2019;108:10-6. [CrossRef]

6. Buchwald H, Avidor Y, Braunwald E, Jensen MD, Pories W, Fahrbach K, et al. Bariatric surgery: a systematic review and meta-analysis. JAMA 2004;292:1724-37. [CrossRef]

7. Kehagias I, Zygomalas A, Karavias D, Karamanakos S. Sleeve gastrectomy: have we finally found the holy grail of bariatric surgery? A review of the literature. Eur Rev Med Pharmacol Sci 2016;20:4930-42.

8. Pech N, Meyer F, Lippert H, Manger T, Stroh C. Complications, reoperations, and nutrient deficiencies two years after sleeve gastrectomy. J Obes 2012;2012:828737. [CrossRef]

9. Braghetto I, Korn O, Valladares H, Gutiérrez L, Csendes A, Debandi $A$, et al. Laparoscopic sleeve gastrectomy: surgical technique, indications and clinical results. Obes Surg 2007; 17:1442-50. [CrossRef]

10. ten Broeke R, Bravenboer B, Smulders FJ. Iron deficiency before and after bariatric surgery: the need for iron supplementation. Neth J Med 2013;71:412-7.

11. World Health Organization (2011). Haemoglobin concentrations for the diagnosis of anaemia and assessment of severity. Vitamin and Mineral Nutrition Information System. Available at: http://www.who.int/vmnis/indicators/haemoglobin. pdf. Accessed Mar 27, 2020.

12. Skikne BS, Lynch SR, Cook JD. Role of gastric acid in food iron absorption. Gastroenterology 1981;81:1068-71.

13. Ruz M, Carrasco F, Rojas P, Codoceo J, Inostroza J, Basfi-Fer $\mathrm{K}$, et al. Heme- and nonheme-iron absorption and iron status 12 mo after sleeve gastrectomy and Roux-en-Y gastric bypass in morbidly obese women. Am J Clin Nutr 2012;96:8107. [CrossRef]

14. Amini M, Zare A, Sobhani Z, Hashemi SS, Vafa L. The Effect of Laparoscopic Sleeve Gastrectomy on Serum Iron Level. Int j of Nutr Sci 2018;3:212-5.

15. Steenackers N, Van der Schueren B, Mertens A, Lannoo $M$, Grauwet T, Augustijns $P$, et al. Iron deficiency after bariatric surgery: what is the real problem? Proc Nutr Soc 2018;77:445-55. [CrossRef]

16. Tussing-Humphreys L, Pini M, Ponemone V, Braunschweig $C$, Fantuzzi G. Suppressed cytokine production in whole blood cultures may be related to iron status and hepcidin and is partially corrected following weight reduction in morbidly obese pre-menopausal women. Cytokine 2011;53:201-6.

17. McClung JP, Karl JP. Iron deficiency and obesity: the contribution of inflammation and diminished iron absorption. Nutr Rev 2009;67:100-4. [CrossRef] 